\title{
LA MÉCANIQUE DES FLUIDES AUX TRÈS BASSES TEMPÉRATURES
}

\author{
Séance organisée le Vendredi 16 mars 1962 par la Socnéts Hyonotechnique de France \\ et la Société fraxçaise de Physique, sous la présidence de M. Yvon, \\ Directeur au Commissariat à l'Énergie Atomique.
}

\section{ALLOCUTION DE IM. YVON}

Nous allons ouvrir la séance. Avant de donner la parole à nos éminents conférenciers, je voudrais critiquer le titre de la séance. Vous trouverez que je ne suis pas très courtois vis-à-vis du Comité Technique de la Société Hydrotechnique de France et de son Président. Mais peut-être puurrais-je atténuer la virulence de ces premiers mots en remarquant d'abord que les critiques s'adressent plus à l'usage qu'au Comité lui-même, et qu'ensuite ces critiques ne sont pas constructives, si bien que ce sont des criticues pas tellement insupportables à entendre. Si vous voulez, c'est une maniere d'analyser le sujet.

Dans le titre "La Mécanique des fluides aux très basses températures ", je voudrais d'abord insister sur l'aspect " très basses températures ". Naturellement la Société Hydrotechnique ne s'est rien refusé en ce domaine : il s'agit des températures les plus basses possibles. Les températures étant les plus basses possibles, on sait bien qu'alors tout est congelé, sauf peut-ètre quelques timides vapeurs qui peuvent encore flotter, mais dont les propriétés physiques ne sont pas d'un très grand intérêt.

Il y a toutefois un liquide qu'on ne peut pas congeler, c'est l'hélium. Done, le sujet de cette réunion ne peut pas être: les propriétés mécaniques des fluides aux très basses températures, mais seulement les propriétés d'un certain liquide aux très basses températures.

Ce problème vaut la peine d'ètre examiné parce que l'hélium présente des propriétés inattendues.

D'abord, il résiste à la solidification. Il reste liquide jusqu'au zéro absolu. On ne peut le solidifier qu'en. exerçant sur lui une pression qui dépasse 25 atmosspheres. En admettant que la pression reste au voisinage de l'ambiance, l'hélium reste liquide.

De plus, il présente des propriétés thermodynamiques très curieuses : ses propriétés de chaleur de transformation et aussi sa chaleur spécifique méritent l'attention, ces phénomènes se déroulant en-dessous d'une température qui est de 2,2 degrés absolus. Aussi distingue-t-on l'hélium II en-dessous de 2,2 degrés et l'hélium I au-dessus. On constate tout de même qu'un certain nombre de lois de la physique ne sont pas tellement détruites. En particulier, la formule de Clapeyron qui est bien connue en thermodynamique résiste à l'épreuve des faits : c'est encore une très bonne formule.

Si au lieu de s'occuper des phénomènes d'équilibre, on s'occupe des phénomènes d'écoulement, la situation est encore plus originale : on constate des phénomènes extraordinaires à propos de la viscosité, à propos des films que l'hélium étend à la surface des solides.
Et quand on fait intervenir des différences de température, les effets thermomécaniques (effet Fontaine) sont extraordinaires.

Nous pourrions maintenant changer le titre de notre réunion, et dire que les conférenciers vont parler de la thermomécanique d'un liquide à très basse température.

Naturellement, un aspect interessant de ces questions, c'est d'essayer de comprendre les phénomènes et par consécuent d'en présenter une interprétation théorique ou de tenter d'en présenter une.

Ces théories reposent sur deux idées essentielles. La première, c'est que e'est de la physique quantique. Le premier principe indispensable est le principe d'incertitude; et le second, c'est le fait que les atomes d'hélium, qui sont en même temps les molécules puisque l'hélium est monoatomique, sont des bosons. C'est là-dessus que se basent les interprétations.

Les bosons me font penser à temps que l'hélium a deux isotopes: l'hélium de masse 4 et l'hélium de masse 3 . En pratique, on a affaire à l'hélium de masse 4 . qui est constitué par des bosons, l'hélium de masse 3 est constitué par des fermions.

Les phénomènes qui nous intéressent aujourd'hui ne concernent que l'hélium 4. Mais comme l'hélium 3 reste liquide lui aussi aux basses températures, et comme on peut mélanger ces deux isotopes en toute proportion, le titre de la réunion peut s'élargir un peu à nouveau : après avoir dit que nous parlerions de la mécanique d'un liquide, nous pourrions maintenant revenir à la mécanique de liquides.

Je voudrais encore que nous nous posions une question avant de donner la parole aux conférenciers: est-ce que l'eau est un liquide quantique?

Nous venons de dire que pour interpreter les propriétés de cet hélium liquide aux très basses températures, il fallait faire appel aux demières ressources de la science. Eh bien! on peut se demander si l'eau, liquide assez connu ici, pent constituer un liquide quantique? Oui, puisque l'existence des molécules est un phénomène quantique. La structure de l'eau à son origine est vraiment quelque chose qui relève de la physique quantique. Quant à savoir comment les molécules de l'eau glissent les unes par rapport aux autres, c'est beaucoup moins frappant, et l'ensemble des propriétés de l'eau ne manifeste pas tellement de caractères quantiques. Mais la structure intime de l'eau est certainement quantique. Il en est d'ailleurs ainsi de tous les phénomènes naturels. 


\section{GÉNÉRALITÉS SUR L'HÉLIUM SUPERFLUIDE PROBLEMIE DE LA VITESSE CRITIQUE}

Par L. WeIL

Doyen de la Faculté des Sciences de Grenoble.

M. le Doyen Weil n'a pas eu, malheureusement, le temps de rédiger le remarquable exposé qu'il a fait en sêance; nous le regrettons vivement et nous nous bornons, avec son accord, à donner ci-après le rêsumé de son mémoire, en français et en anglais et la bibliographie qu'il a bien voulu nous indiquer sur la question.

Résumé. - On interprète en général les expériences de superfluidité dans l'hélium 2 par l'hypothèse de l'existence de deux fluides, l'un normal, l'autre superfluide.

Ces deux fluides sont caractérisés par un certain nombre d'expériences, dont l'expérience d'Andronikachvili. Il s'agit là d'un simple modèle; la réalité est bien plus complexe, puisque en vérité on a affaire à un fluide de BoseEinstein dégénéré, et la séparation en deux fluides est arbitraire; il s'agit simplement, quand on parle de fluide normal, d'un ensemble de perturbations du liquide de BoseEinstein, à l'état fondamental au zéro absolu seulement. On comprend, dans cette optique, que les phénomènes hydrodynamiques anormaux ne se produisent que jusqu'à des vitesses d'écoulement fort limitées; on discute les causes qui agissent pour limiter cette vitesse critique (théorie de Landau, theorie de Feynmann).

Abstract. - In general one interprets the experiments on the superfluid properties of helium II on the hypothesis that, at the very low temperatures in question, the liquid behaves as a mixture of two fluids the one normal and the other superfluid.

The characteristics of these two fluids have been determined from a number of experiments, notably of Andronikachvili. The two fluid model is a simplification; the real liquid is much more complex since it is in fact a degenerate Bose-Einstein fluid whose separation into two components is arbitrary. When one speaks of the normal fluid one is, to be more exact, referring to a collection of disturbances in the Bose-Einstein fluid which itself remains in its ground state, as if it were at the absolute zero. Seen from this angle therefore the anomalous hydrostatic phenomena only occur up to very limited values of the flow velocity. The factors which limit this critical flow velocity, will be discussed, with reference in particular to the theories of Landau and Feynmann.

\section{BIBLIOGRAPHIE}

I. - Progress in Low temperature Physics, Editor C. J. Gorter, North Holland Publ Gic (Amsterdam). Volumes I, II, III.

II. - Supplements to helium par Lipscirtz et ANoroNikachvir, Éditor : Consultants Bureau, NewYork, 1959.

III. - Liquid helium. Atrinis (K. R.), Cambridge University Press.

Nota : I et III sont donnés, aussi, dans la bibliographie du mémoire de M. Kramers.

\section{SUR LA THÉORIE DE L'ÉTAT SUPERFLUIDE}

Par M. Jacques Winter, Examinateur à l'École Polytechnique,

Résumé. - On expose une interprétation de la théorie de London: Le superfluide serait constitué d'atomes d'hélium délocalisés ảont les fonctions d'ondes coïncide- raient. On énumère les faits que cette théorie interprète aisément, absence de viscosité, effet thermomécanique, et surtout la vitesse limite d'écoulement des films de Rollin.

Abstract. - We give an interpretation of London's theory. The superfluid should be formed of unlocalized helium atoms, with the same wave functions. Facts which this theory can explain easily are listed : Absence of viscosity, thermomechanical effect, and, especially, the critical speed of Rollin films.

Des diverses interprétations des phénomènes de superfluidité, aucune ne nous paraît satisfaisante. Les unes, basées sur les idées de M. Landau et le concept de roton (rotation quantifiée d'un ensemble d'atomes d'hélium II) conviennent au voisinage du zéro absolu, mais ne rendent pas compte des phénomènes de la transition $\lambda$ (à 2018 absolu, sous la pression atmosphérique), les autres basées sur les idées de M. London, qui assimilent l'hélium II à un gaz de Bose-Einstein, cherchent à expliquer la transition $\lambda$ et les phénomènes an voisinage de 2018 , mais conviennent mal aux très basses températures, voisines de $00^{\circ}$.

I. Idée fondamentale de M. London. - La superfluidité n'existe pas pour l'isotope ${ }^{3}$ He qui est un fermion. Donc, ces propriétés sont liées aux caractères du boson. Écrivons la loi de distribution statistique de $N$ bosons, à la température $T(\beta=1 / k T)$ sur des niveaux d'énergie $\varepsilon_{i}, g_{i}$ étant la multiplicité de chaque niveau.

$$
N=\sum N_{\mathrm{i}}=\sum_{\mathrm{i}} \frac{g_{\mathrm{i}}}{e^{\beta \varepsilon_{\mathrm{i}}+\alpha}-1} .
$$

$\alpha$ est une constante que l'équation (1) doit permettre de déterminer. Elle doit être positive, sinon certains des $N_{i}$ seraient négatifs (pour $\varepsilon_{i}<-\alpha / \beta$ ), et est proportionnelle au potentiel de Gibbs.

Si l'on considère des particules sans spin, de masse $M$, dans un volume $V$ on admet en général :

$$
s_{p}=p^{2} / 2 M \quad g(p) \mathrm{d} p=4 \pi\left(V / h^{3}\right) p^{2} \mathrm{~d} p .
$$

$p$ étant la quantité de mouvement. En remplaçant la somme de (1) par une intégrale:

$N=\frac{4_{4} \pi V}{h^{3}} \int_{0}^{\infty} \frac{p^{2} \mathrm{~d} p}{\mathrm{e}^{\alpha+\beta} p^{2} / 2 M-1}=\frac{V}{h^{3}}(2 \pi M k T)^{3 / 2} F(\alpha)$,

avec

$$
\mathrm{F}(\alpha)=\frac{2}{\pi^{1 / 2}} \int_{0}^{\infty} \frac{Z^{1 / 2} \mathrm{~d} Z}{\mathrm{e}^{\alpha+Z}-1}
$$

pour $\alpha=0, F=2,612$ et décroît pour $\alpha>0$.

Pour

$$
T<T_{0}, T_{0}=\left(\frac{N}{2,612 V}\right)^{2 / 3} \frac{h^{2}}{2 \pi M K}=3^{\circ}, 13 .
$$

l'équation (2) ne permet plus de calculer $\alpha$. Mais, bien évidemment (1) doit toujours avoir une racine en $\alpha$, avec valeurs positives des $N_{i}$. En effet, le remplacement de la somme par l'intégrale, pour de très petites valeurs de $T$, explique cette contradiction. M. London a séparé un terme, le premier de la somme et a discutél'équation ainsi obtenue. Il a cru mettre en évidence une discontinuité dans les dérivées des fonctions thermodynamiques par rapport à la température 
au point $T=T_{0}$, et a vu là, l'origine du point $\lambda$, passage à la superfluidité. C'était une erreur. L'équation (1) n'introduit aucune discontinuité et c'est au croisement de 2 expressions approchées de la fonction $N$ qu'on a cru voir une discontinuité.

Telle qu'elle était exposée dans son mémoire de la Physical Review, de 1938 (t. 54, p. 747) cette théorie était erronée, mais la nature de fermion de l'hélium intervient certainement. L'explication du point $\lambda$ en assimilant l'hélium II a un solide parait vraiment paradoxale et nous avons cherché à réexprimer autrement la théorie de M. London.

II. Nouvelle expression de cette théorie. - Est-il possible de prendre littéralement l'expression "gaz de Bose-Einstein "? Autrement dit, est-il possible de considérer l'Hélium II comme un ensemble d'atomes délocalisés ou étalés dans l'espace, comme ceux d'un gaz dans l'interprétation de la mécanique quantique? Ou devons-nous les considérer comme localisés, ce qui est le cas d'un liquide ?

Il est habituel de considérer l'hélium II comme un mélange de deux liquides de densités $\rho_{n}$ (normal) et $\rho_{s}$ (superfluide), en suivant les idées de $M$. Tisza. Mais on ne peut pas définir deux espèces différentes d'atomes d'hélium. On admet donc que les quanta d'excitation, de vibrations et rotations d'ensemble, se comportent comme des fluides et que ce sont les quanta de rotation (rotons) qui constituent le fluide normal. Mais il est difficile de comprendre ce qui arrive aux rotons à $T=2,18^{\circ}$, ni pourquoi ils s'écartent des parois des tubes fins, tout en adhérant aux parois des disques en rotation.

Dans quelles conditions peut-on considérer l'hélium comme un gaz ? Si l'on veut calculer les ondes propres, associées aux atomes de He, par la mécanique quantique, il faut faire intervenir l'interaction coulombienne $J$ des deux atomes; elle donnera un potentiel répulsif important pour des distances $\left|R_{1}-R_{2}\right|<2$ Angstroms environ, $R_{1}$ et $R_{2}$ étant les rayons vecteurs des noyaux, en fonction desquelles s'exprimeront les $\psi$ des deux atomes. Si cet espace de recouvrement est une fraction négligeable du volume total, l'énergie d'interaction :

$$
\begin{aligned}
& \int \psi_{1}^{*} \psi_{1} J \psi_{2}^{*} \psi_{2} \mathrm{~d} V_{1} \mathrm{~d} V_{2} \mid \int \psi_{1}^{*} \psi_{1} \psi_{2}^{*} \psi_{2} \mathrm{~d} V_{1} \mathrm{~d} V_{2}, \\
& \left|R_{1}-R_{2}\right|<2 \AA
\end{aligned}
$$

sera négligeable, mais s'il y a $N$ atomes, il faudra combiner tous les atomes 2 à 2 et l'interaction ne sera négligeable que si le remplissage total du volume $V$ est négligeable. Pour le cas de l'hélium $I I$, sa densité est 0,14 . Même en prenant un remplissage de l'ordre de $1 / 100$, cela divisera l'énergie $J$ par $10^{4}$, ce qui donnera des énergies de plusieurs centièmes d'électronvolts, par atome, en admettant que l'énergie de répulsion soit de l'ordre de quelques dizaines d'électronvolts (la répulsion des 2 électrons de l'hélium est de l'ordre de 4 à 5 électron-volts). Cette énergie de répulsion est beaucoup trop forte pour le but que nous recherchons. Il y a donc là une grosse objection à cette théorie, à laquelle nous répondons à la fin du paragraphe III.

Reprenons done l'examen de notre exposé de 1958 ( $\left.{ }^{1}\right)$.

(') Journal de Physique, 1958, 19, 532.
III. - Modèle mathématique. - On suppose que l'atome d'hélium se déplace dans un potentiel périodique, dont on ne considerera pour simplifier qu'une dimension. Soit $\mathrm{N}$ le nombre de périodes. Ce champ périodique sera constitué par l'ensemble des autres atomes d'hélium dont il représentera l'interaction moyenne. Soit $E_{0}$ l'énergie de cet atome supposé renfermé, dans une seule période. $E_{0}$ sera une valeur propre du champ d'une seule cuvette de potentiel. Si $U$ est la heuteur de la cuvette, l'atome aura nne quantité de mouvement $p$ donnée par :

$$
p^{2}=2 M(E-U) \text {. }
$$

( $M$, masse de l'atome). Si l'on juxtapose les $N$ cuvettes, on cherche ce que devient la fonction propre et la valeur propre $E_{0}$ d'une seule cuvette. On suppose la quantité de mouvement très lentement variable dans le champ et pour les applications numériques, on remplace les cuvettes par un potentiel en créneau. Ce qui permet de remplacer $|p|$ par une valeur moyenne $p=\sqrt{2 M H}(H$, valeur moyenne de $E-U)$.

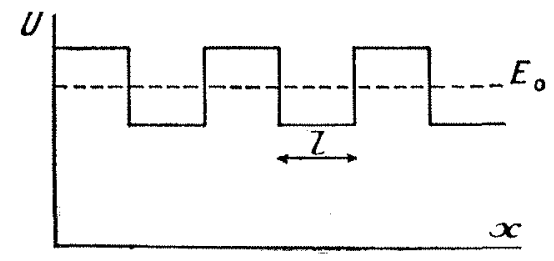

FIG. 1.

Alors, le niveau $E_{0}$ est coupé en $N$ niveaux voisins. Ceci correspond au fait général qu'à un domaine plus étendu (les domaines de propagation des ondes étant assimilés aux fonds de cuvette) correspondent plus de valeurs propres; à la limite, pour de grands domaines, le nombre des niveaux étant proportionnels aux domaines. Les uns sont déplacés vers le haut et les autres vers le bas; ils sont équidistants, et le niveau moyen n'est pas déplacé. Mais le niveau le plus bas est déplacé par rapport à $E_{0}$ d'une quantité

$$
\Delta E=\pi \frac{\bar{p}}{l M} \mathrm{e}^{-\eta \bar{\nu} / \hbar}
$$

$\hbar=h / 2 \pi, l$, longueur d'une $1 / 2$ période. Ce calcul est très grossier. L'adoption d'un potentiel rectiligne est rajoutée à la fin pour simplifier l'estimation des valeurs numériques.

$$
\begin{gathered}
\text { Pour } H=1 / 3200 \mathrm{eV}, l=2 \AA \text {, on a } \\
\Delta E=1,410^{-4} \mathrm{eV} .
\end{gathered}
$$

Or, si l'on estime la valeur $T S, S$ entropie de l'hélium au point $\lambda$ que l'on peut mesurer expérimentalement, d'après les expériences de Kapitza, on trouve la même valeur. Il n'est donc pas absurde d'envisager la superfluidisation comme un changement d'état, entratnant une diminution d'énergie $\Delta E$ et une disparition d'entropie, les énergies libres $E-T S$ restant les mêmes au point $\lambda$. Comme la superfluidisation entraine une légère dilatation, il eût été correct d'écrire :

$$
\begin{aligned}
& T S=E_{n}-E_{s}+P\left(V_{n}-V_{s}\right) \\
& T S=\Delta E-P \Delta V \\
& \left(V_{1}, V_{\mathrm{s}} \text { volumes spécifiques }\right)
\end{aligned}
$$


$P$ pression. Ceci suppose le volume d'ensemble variable et les pressions et températures données. Mais pour connaitre $\Delta V$, il faut connaitre la proportion exacte de superfluide.

Tout ceci présente donc un caractère qualificatif et hypothétique et l'on voit, comme $\Delta E \sim 10^{-4} \mathrm{eV}$, qu'il faut que l'effet de la répulsion coulombienne des charges d'hélium, de l'ordre des dixièmes de $\mathrm{eV}$, soit éliminé.

Supposons que l'étalement mette en cause un nombre d'atomes beaucoup plus grand que 1000 et le phénomène n'a de sens que dans ce cas. Soit par exemple $10^{8}$. Le niveau énergétique fondamental localisé sera coupé, comme nous l'avons dit en $10^{8}$ composantes, la moitié activée, la moitié rabajssée. Distribuons les atomes sur les 1000 niveaux les plus stables. La répulsion des atomes, placés sur le même niveau, qui sera étalé dans tout le volume $V$, vaudra celle de 2 atomes dans une case du réseau semi-cristallin $\times\left(10^{5}\right)^{2} /\left(10^{8}\right)^{2}$, pour tenir compte de toutes les attractions 2 à 2 , et de l'étalement (au carré) du volume $V$. Elle sera, très inférieure à $10^{-3} \mathrm{eV}$ (de l'ordre de $10^{-8} \mathrm{eV}$ ) et n'interviendra plus. Quant à l'interaction des atomes de niveaux différents, leurs ondes doivent ètre orthogonales, donc décalées dans l'espace ; les répulsions qui sont dues aux recouvrements seront très diminuées et pourront être compensées par l'effet VAN DER WAALS, attractif, qui se produit pour des distances supérieures à $2 \AA$. Ces considérations sont très grossières, mais un calcul paraît impossible.

Quant àlastabilisation globale elle sera un peu réduite, mais comme les niveaux sont distribués d'une manière uniforme autour du niveau fondamental perturbé, elle ne sera réduite que de $2.10^{-5}$ environ, c'est-à-dire d'une valeur négligeable. L'objection du recouvrement qui serait dirimante pour quelques centaines d'atomes, s'évanouit donc pour $10^{7}$ ou $10^{8}$ atomes. Cette distribution sur des solutions orthogonales les unes aux autres pourra redonner pour la densité globale une structure périodique, qui est effectivement observée. Le niveau fondamental seul donnant une structure uniforme.

On voit que la superfluidisation ne se produira que pour les bosons, les fermions devant se distribuer sur tous les niveaux provenant de la coupure de $E_{0}$ et la valeur moyenne de l'énergie restera inchangée. Il n'y aura dono plus d'effet d'énergie, tendant à la superfluidisation. On voit aussi que si $\mathrm{P}$ est assez grand, d'après (4), l'effet disparaitra ce qui est conforme à l'expérience.

IV. Applications. - Il reste à montrer que cette théorie de l'étalement des ondes, dont les faiblesses ne nous échappent pas, rend compte des phénomènes paradoxaux de l'hélium $I I$.

A. Disparition de l'entropie du superfluide. - Nous avons déjà utilisé ce fait dans le calcul du $\S$ III, écrivant $S_{n}$ pour $\Delta S$. Le remplacement d'un ensemble d'atomes localisés, par un ensemble délocalisé entraine une grosse diminution d'entropie, à température suffisamment basse. La présence d'un seul phonon sur $N$ atomes localisés, donne $N$ niveaux soit une entropie $K \log N$, et un seul niveau s'ils sont délocalisés. Cette question soulève une difficulté, signalée dans notre article cité.

B. Disparition de la viscosité. - La viscosité sur les parois est due à une interaction des molécules de la paroi et des molécules du fluide. Si celles-ci sont délocalisées ou étalées, l'interaction est diminuée en proportion. De même, à l'intérieur d'une masse délocalisée, on ne peut plus définir d'attraction entre deux atomes voisins et, donc, plus de viscosité.

C. Erfet rontaine ou force thermique. Pourquoi l'atome superfluide est-il attiré vers les zônes chaudes de l'hélium, ce qui caractérise l'effet thermomécanique? Dans une expérience d'effet fontaine, considérons les atomes superfluides, qui passent du récipient froid au récipient chauffé. Une dépression doit exister du côté chauffé. Quelle est son origine? Une attraction crée par les rotons, ou le rayonnement électromagnétique ? Une telle force n'est pas expliquée. Avec les hypothèses faites, un atome peut se relocaliser dans une zone chauffée, ce qui provoquera un déplacement comme dans toute transition quantique, et un vide au voisinage de la jonction des récipients, d'où une dépression. L'abondance des quanta d'énergie dans la zône chauffée provoquera les relocalisations.

D. Films superficiels, - Pourquoi le superfluide vient-il se placer au voisinage des parois, ce qui créée tous les phénomènes dûs aux films de Rollin ? Au voisinage d'une paroi fixe, les molécules fixes font régner un champ électrostatique qui limite le nombre des états propres possibles des atomes d'hélium. Donc l'entropie $S_{n}$ à température donnée, décroît au voisinage des parois. Done, la superfluidisation se produira plus facilement dans la couche limite puisque $S_{n}$ est le terme qui l'empêche. Il y aura donc une couche de superfluide au voisinage des parois fixes, qui constituera les films de Rollin, et explique les expériences de passage par les ouvertures capillaires.

E. VItesses limites D'ÉCOULEMENT DES FHams superficress. - Ces vitesses sont de l'ordre de $50 \mathrm{~cm}$ à $1 \mathrm{~m}$ seconde et sont trop faibles pour que les énergies cinétiques du courant puissent provoquer une relocalisation et une rupture du film d'hélium. Il s'agit là de l'effet le plus délicat à expliquer. Ce que nous avons dit à ce sujet (dernier paragraphe de notre article cité) doit ètre annulé : il faut porter son attention non pas sur le terme $\Delta E$, mais sur le terme $T S_{\mathrm{n}}$.

Quelle est la durée moyenne du séjour d'un atome d'hélium normal dans son volume du réseau liquide, dans sa case, dirons-nous ? Posons : (1)

$$
\tau=\tau_{0} e^{\frac{H}{k T}} \text {. }
$$

$\tau_{0}$ est la durée obtenue en divisant par sa vitesse $\sqrt{k T / M}=v$, la largeur de la case, $2 \AA$ environ.

$$
v=\sqrt{\frac{1,38 \cdot 10^{-16} \cdot 2,2}{3,34 \cdot 10^{-24}}}=10^{4} .
$$

1. Voir FRENKEL, Introduction à la théorie des métaux (p. 256, édition russe). 
Done

$$
\tau_{0}=2 \cdot 10^{-12} \text {, }
$$

$H$ hauteur de la barrière de potentiel limitant la case est prise égale à $1 / 3200 \mathrm{eV}=5.10^{-16}$ C. G. S. Mais il faut doubler ce chiffre car c'est la hauteur totale de la barrière qu'il faut prendre ici et non la valeur moyenne de $\left(p^{2} / 2 M\right)$. De plus, $k T \approx 2,810^{-16}$.

On a done: $\tau \sim 2.10^{-12} \mathrm{e}^{4} \sim 10^{-10} \mathrm{~s}$.

Pour des vitesses relatives liquide-paroi de l'ordre de $50-100 \mathrm{~cm} / \mathrm{s}$, nous voyons qu'une molécule de la paroi traverse la largeur $2 \AA=2.10^{-8} \mathrm{~cm}$ dans un temps de l'ordre de $2.10^{-10} \mathrm{~s}$.

Le champ électrostatique qui s'exercera sur un atome d'hélium localisé et qui le bloque, varie pendant un intervalle de temps de l'ordre de la durée de séjour moyen de l'atome dans son volume élémentajre, dans sa case. L'effet ordonnateur disparait, $S$ augmente et les atomes délocalisés se relocalisent, en vertu des lois de la mécanique statistique. L'apparition et la disparition du film superfluide sont donc essentiellement des effets d'entropie et non d'énergie.

V. Vérifications. - Est-il possible de vérifier cette théorie directement, autrement que par ses conséquences, décrites ci-dessus ?

a) Des expériences de diffraction, qui étudient directement la structure du liquide semblent être le moyen le plus indiqué. Mais la théorie délocalisée laisse subsister nne structure du potentiel moyen; ce point a été admis par nous, plus que démontré. Cette structure serait irrégulière comme celle d'un liquide et le moyen de faire une distinction paraît difficile, en l'état actuel de la théorie délocalisée.

b) L'existence du mouvement brownien semble être le meilleur critère. Si l'on peut immerger dans l'hélium des particules collö̈dales aussi petites que possible, le mouvement brownien doit subir une décroissance subite au point $\lambda$, lorsque la température décroît et doit pratiquement disparaître dès qu'on s'est écarté de la température $\lambda$, dans la théorie délocalisée, puisque l'onde délocalisée enveloppe la particule et que les chocs, à la base du mouvement brownien n'ont plus de raison de se produire.

\section{Discussion}

M. Chapouthier demande à M. Winter dans quelle mesure la théorie de l'hélium superfluide explique l'effet Prandtl de nullité de vitesse à la paroi pour les fluides courants : c'est un dogme classiqueen hydraulique que de dire qu'il n'y a pas de glissement d'un fluide en contact de l'obstacle, or, M. Winter a montré que, dans certains cas, il peut y en avoir.

M. Winter n'a vu aucun ouvrage donnant une théorie physique de l'effet Prandtl, mais il pense que le dogme est valable pour un fluide normal à atomes localisés : entre les atomes du liquide et ceux de la paroi, il est probable qu'il y a une attraction qui explique que le liquide reste en contact, c'est le blocage de la vitesse, qui est constaté expérimentalement pour un liquide et même pour un gaz (effet Prandtl autour d'une hélice d'avion).

Mais cet effet disparaît pour le superfluide parce que l'atome du superfluide est étalé sur $10^{4}$ fois la largeur de l'atome de la paroi. A partir du moment oú l'atome se reconcentre et oi il n'est plus superfluide, l'effet Prandtl reparait et l'atome se bloque.

M. le Président ajoute que c'est simplement le choc sur les parois qui, pour les gaz, ramene les vitesses dans tous les sens. Pour les liquides, c'est plus mystérieux, car on n'a pas de moyen d'analyse précis.

M. le Président demande des explications sur les courbes de London et le livre de Sommerfeld.

M. Winter répond qu'il n'y a pas de courbes dans le livre de Sommerfeld, mais que la courbe de London se trouve dans le mémoire de celui-ci et qu'elle est reproduite dans une quinzaine de traités de physique théorique, aussi bien américains que soviétiques. Or, d'après M. Rideau, Maître de recherches au C. N. R. S., Américains et Soviétiques se seraient trouvés d'accord pour reconnaître l'inexactitude de cette courbe dans le cas d'un très grand domaine grâce à des calculs faits par machine électronique. Mais, au point de vue de M. Winter, l'allure de l'équation donnant la constante (alpha) correspond à une équation régulière et à une série rapidement convergente, et on ne voit pas très bien comment une discontinuité pourrait s'établir.

M. le Président rappelle que, d'après l'exposé de M. Winter, la paroi et ses aspérités peuvent avoir un effet sur la vitesse tangentielle et demande à M. Kramers s'il a connaissance d'expériences mettant en évidence des phénomènes de ce genre.

M. Kramers fait un signe négatif et indique qu'on n'est pas très sûr de l'existence de la vitesse limite.

M. Winter ajoute que seules des expériences de mouvements browniens permettraient de vérifier directement la théorie qu'il a exposée.

M. le Président rappelle que Careri, en Italie, fait une série d'expériences intéressantes sur la diffusion des particules chargées dans l'hélium.

M. le Président remercie vivement M. Winter.

\section{QUELQUES EXPERIENCES SUR LA TURBULENCE ET LA FRICTION MUTUELLE DANS L'HÉLIUM LIQUIDE}

Par M. H. C. Kramers, Kamerlingh Onnes Laboratorium, Leyde.

Résumé. - Discussion des expériences récentes sur les phénomènes hydrodynamiques de l'hélium liquide, exécutées au Laboratoire Kamerlingh Onnes à Leyde.

Rappel des recherches de M. Staas et M. Taconis sur les mesures des gradients de la température et de la pression d'un courant continu dans un tube étroit de section circulaire. Un phénomène de turbulence, presque entièrement identique à la turbulence dans un liquide classique, était établi.

Expériences exécutées par M. Wiarda et l'auteur sur l'étude de l'atténuation du second son en fonction d'un courant continu simultané. La méthode du second son se montre très convenable pour l'investigation des phénomène qu'on attribue à la friction mutuelle.

Abstract. - Recent experiments on the hydrodynamical properties of liquid helium II, performed in the Kamerlingh 
Onnes Laboratorium (Leiden) are discussed on the basis of the two-fluid model.

Staas and Taconis measured temperature and pressure gradients occuring in a narrow circular capillary in the presence of a heat current. They discovered a turbulent phenomenon, very analogous to ordinary classical turbulence.

The attenuation of second sound was studied by Wiarda and the present author in its dependence on a simultaneous continuous heat current. The method employed proves to be very suitable for a study of the so-called mutual friction and of phenomena occurring in the critical velocity region.

\section{TABLE DES SYMBOLES UTILISES}

$T \quad$ La température, $p$ la pression.

$S \quad$ L'entropie spécificque

$W \quad$ La densité de flux de chaleur.

$v_{n}, v_{s}, w$ La vélocité normale, superfluide et relative.

$\rho_{n}, \rho_{s}, \rho$ La densité normale, superfluide et totale.

$\eta_{n} \quad$ La viscosité normale.

$v_{\text {II }} \quad$ La vélocité du second son.

$r \quad$ Le rayon d'un tube circulaire.

$l \quad$ La longueur d'un tube.

V Le volume.

$\vee \quad$ La fréquence du second son.

A Le coefficient du frottement mutuel.

Introduction. - Liobjet de ce mémoire est la présentation de quelques recherches sur l'hydrodynamique de l'hélium II. Il s'agit de recherches expérimentales sur les phénomènes qu'on rencontre dans l'écoulement du liquide par un tube de section circulaire.

Précisons dès le début de cette séance qu'on voudra utiliser une interprétation de base: I'hypothèse ou plutôt le modèle des deux fluides. Résumons encore une fois les propriétés de ce modèle.

Le liquide est caractérisé comme étant composé de deux fluides inséparables : le fluide normal se comporte comme un fluide visqueux ordinaire et le superfluide, lequel ne souffrant pas de friction des parois, se conduit comme unfluide idéal Tous les deux fluides possèdent leurs propres vélocités et densités, la somme des deux densités étant égale à la densité totale du liquide. Au point de transition le rapport $p_{1} / 0$ est identique à l'unité, au zéro absolu il n'existe plus de fluide normal. Ce rapport décroît rapidement lorsqu'on diminue la température à partir du poiut de transition.

L'énergie et l'entropie du liquide sont liés au fluide normal exclusivement. Ainsi, le transport de chaleur est éffectué par la convection de celui-ci et on a pour la densité de flux de chaleur:

$$
W=\rho S T v_{\mathrm{n}} \text {. }
$$

Une vraie conductibilité thermique existant pour le fluide normal, peut être négligée dans tous les cas considérés. La validité de la formule (1) suggère l'importance d'une classe d'expériences qu'on peut désigner comme des mesures de la "conductibilité thermique". La figure 1 montre le principe de cette méthode. Un volume isolé est connecté par une conduite au bain d'hélium à température constante. En cas stationnaire la quantité d'énergie par seconde introduite au moyen de l'élément de chauffage et liée directement à la vélocité normale moyenne dans

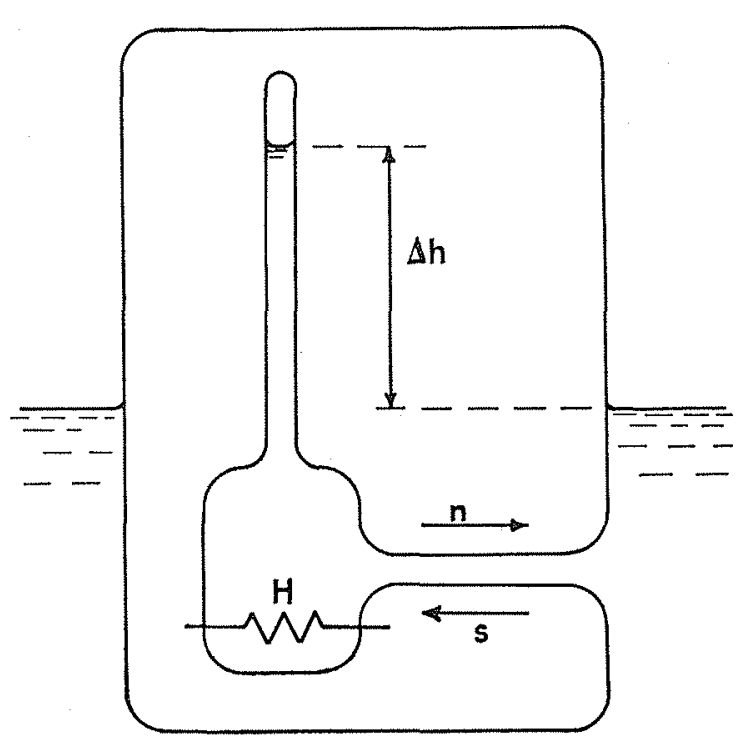

FIG. 1. - La méthode de "la conductibilité thermique ".

la conduite. En outre, parce qu'il n'y a pas de transport net de matière, la vélocité moyenne du superfluide est déterminée par :

$$
\rho_{12} v_{n}+\rho_{\mathrm{s}} v_{\mathrm{s}}=0 \text {. }
$$

Ainsi cette méthode donne la possibilité d'étudier les relations entre les vélocités et les gradients de la température et de la pression. Il faut insister sur le fait que la méthode habituelle des études de l'écoulement isotherme rencontre beaucoup de difficultés expérimentales et, par conséquent, n'est pas convenable pour obtenir des résultats certains.

Les résultats expérimentaux nous forcent de distinguer deux régions de vélocité séparées par une certaine vélocité critique. Cotte vélocité dépend de la température et du diamètre de la conduite. Elle décroît si l'on augmente le diamètre du tube. La situation en région sous-critique est assez simple et s'accorde bien avec les équations du mouvement déduites par Landau et d'autres auteurs sur la base de la théorie des excibations. La région supercritique est plus compliquée, cependant, on peut interpréter les mesures par l'addition d'une force de frottement mutuel aux équations du mouvement (frottement de Gorter et Mellink). Comme nous le verrons, cette force dépend des circonstances et, par consécuent, ne se comporte pas comme un terme propre des équations du mouvement. Néammoins presque toutes les recherches des écoulements stationnaires la mont rent comme proportionnelle au cube de la vélocité relative des deux fluides. Les équations du mouvement peuvent s'écrire:

$$
\begin{aligned}
\rho_{\mathrm{n}} \frac{\partial v_{\mathrm{n}}}{\partial t}+ & \left(v_{\mathrm{n}} \operatorname{grad}\right) v_{\mathrm{n}}=-\frac{\rho_{\mathrm{n}}}{\rho} \operatorname{grad} p \\
& -\rho_{\mathrm{s}} S \operatorname{grad} T+\eta_{\mathrm{n}} \Delta v_{\mathrm{n}}+\left[-A \rho_{\mathrm{n}} \rho_{\mathrm{s}} w^{3}\right] \\
\rho_{\mathrm{s}} \frac{\partial v_{\mathrm{s}}}{\partial t}+\left(v_{\mathrm{s}} \operatorname{grad}\right) v_{\mathrm{s}}= & -\frac{\rho_{\mathrm{s}}}{\rho} \operatorname{grad} p \\
& +\rho_{\mathrm{s}} S \operatorname{grad} T+\left[+A \rho_{\mathrm{n}} \rho_{\mathrm{s}} w^{3}\right]
\end{aligned}
$$

L'analogie de ces équations avec l'équation de 
Navier-Stokes est évidente, seulement le terme en grad $T$ indique les propriétés spéciales de ce modèle. Les termes de frottement mutuel sont posés entre crochets. On doit noter que les termes carrés ne sont pas vérifiés suffisamment, les travaux théoriques sur ce sujet ne s'accordent pas. Quelques termes liés aux phénomènes non-réversibles ont été omis et la compressibilité est négligée. Pour le cas considéré de l'écoulement sans transport net de matière, se limitant d'ailleurs aux tubes de section circulaire, on peut résumer les résultats expérimentaux par les formules:

$$
\begin{gathered}
\operatorname{grad} p=\frac{8 \eta_{\mathrm{n}}}{r^{2}}\left(\frac{\bar{W}}{\rho S T}\right), \\
\rho S \operatorname{grad} T=-\frac{8 \eta_{\mathrm{n}}}{r^{2}}\left(\frac{\bar{W}}{\rho S T}\right)+\left[-A \rho \rho_{\mathrm{n}}\left(\frac{\bar{W}}{\rho S T}\right)^{3}\right]
\end{gathered}
$$

les crochets désignant le terme lié au frottement mutuel, ne se produisant qu'en région supercritique.

La formule (4a) représente la loi de Poiseuille appliquée au fluide normal ; la formule (4b) montre que la même loi régit de la "conductibilité thermique " en région sous-critique.

La relation de H. London pour l'effet fontaine.

$$
\operatorname{grad} p=\rho S \operatorname{grad} T,
$$

n'est valable qu'en région sous-critique.

Découverte d'une turbulence quasi-classique. En principe on peut obtenir la viscosité d'une expérience discutée au-dessus, utilisant la formule $4 a$. Cependant on doit se rappeler que l'applicabilité de cette formule peut ètre limitée par l'influence du libre

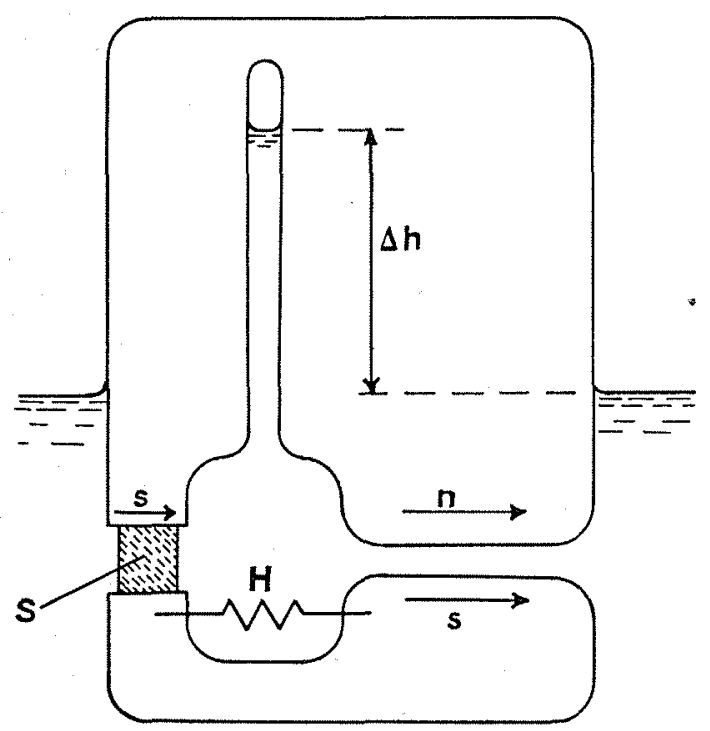

Fig. 2. - La méthode de Taconis et Staas avec l'élimination du frottement mutuel.

parcours et cela signifie pratiquement qu'on ne peut pas utiliser des tubes plus étroits que $50 \mu$ environ. Malheureusement, parce que la vélocité critique est assez petite dans les tubes plus larges, la différence de température s'accroît rapidement pour des vélocités convenables. La grande correction de la pression de vapeur causée par cela, réduit sérieusement la précision de la mesure de la pression.

C'est pour cela que Staas et Taconis ont effectué une modification de l'appareil (fig. 2). $S$ désigne un filtre superfluide (superleak) à travers lequel le fluide normal ne peut pas passer, de mème que la chaleur. Un tel filtre peut être construit à l'aide d'une poudre à polir très fine. Dans l'appareil modifié, le superfluide n'est plus forcé de s'écouler en contre courant au fluide normal comme dans le cas précédent. Au contraire, on peut s'imaginer que les deux fluides auront presque la même vélocité, la différence étant seulement la vélocité relative critique. De cette manière le frottement mutuel est éliminé, ceci est démontré aussi par la validité de la relation de London pour ce cas.

Avant de montrer les résultats on doit se rappeler une interprétation bien connue dans l'hydrodynamique des liquides ordinaires. On pent caractériser la nature de l'écoulement par des grandeurs sans dimension. Considérons l'écoulement dans un tube cylindrique. En régime laminaire on peut écrire la loi de Poiseuille comme une identité entre deux grandeurs sans dimension :

$$
Q=\frac{r^{3}}{4 \eta^{2}} \operatorname{grad} p=\frac{2 r \rho v}{\eta}=R
$$

$R$ étant le nombre de Reynolds. En régime turbulent cette relation n'est plus satisfaite, cependant, les travaux de Blasius ont fourni une formule empirique :

$$
Q=4,94 \times 10^{-3} R^{1.75}
$$

Le résultat surprenant des recherches de Staas et Taconis est la validité des mêmes formules pour le cas de l'hélium; seulement on doit remplacer $v$ par $v_{n}$ et $\eta$ par $\eta_{11}$. On s'attendrait à ce qu'on doive remplacer aussi $p$ par $p_{n}$, parce que la viscosité est liée au fluide normal mais ceci ne donne pas un résultat universel.

Sur la figure 3 on voit des lignes correspondant anx formules (5) et (6) et les points expérimentaux obtenus aux températures diverses entre 1 oK et 2,1 oK et dans des tubes de diamètre 82, 173 et $255 \mu$; l'accord se montre très satisfaisant.

L'examen détaillé fait noter une différence entre l'hélium et un liquide ordinaire. C'est l'absence d'une région de transition entre les régimes laminaire ot turbulent. Les points expérimentaux sont toujours situés sur une des deux lignes droites. L'intersection des lignes se trouve à une valeur de $R$ égal à 1200 . Comme dans le cas ordinaire il se montre possible d'étendre le régime laminaire par une augmentation très graduelle de $r_{\mathrm{n}}$.

La préférence de la nature pour un nombre Reynolds contenant la densité totale au lieu de la densité normale ne semble pas trop singulière dans ce cas. En effet on peut avoir un écoulement de la totalité du liquide. le fluide normal entraînant le superfluide grâce au frottement mutuel. Cependant, l'étude suivante montre que cette condition n'est pas impérative pour obtenir le résultat mentionné.

Malgré les objections notées, il se montre possible d'effectuer quelques expériences au moyen de l'appareil sans filtre. En ce cas le résultat est exactement le 

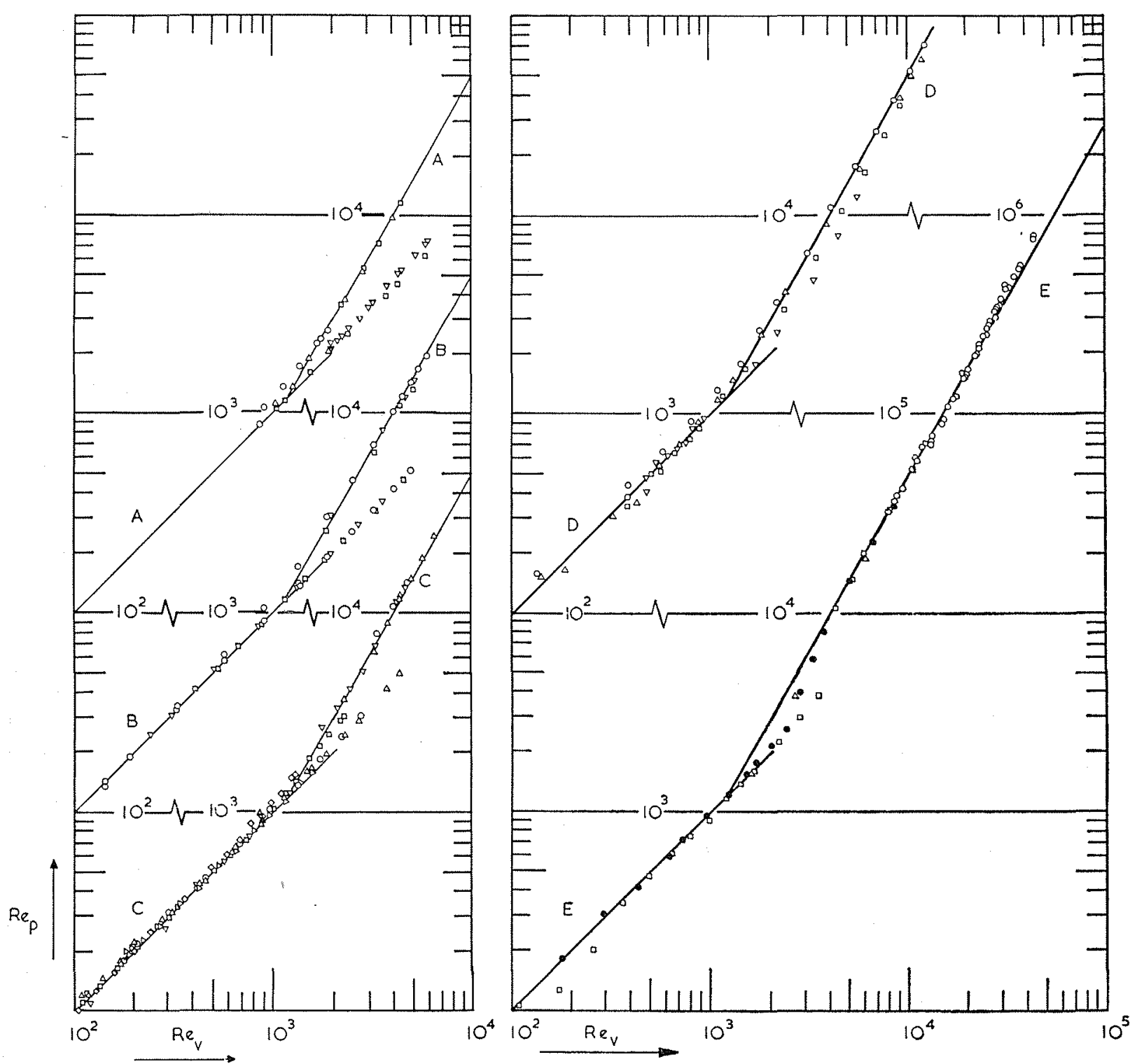

Fig. 3. - Dépendance entre $Q\left(=R e_{p}\right)$ et le nombre de Reynolds (= $R e_{\mathrm{V}}$ ) pour les capillaires du diamètre 173,5 et $225 \mu$.

Résultats de la région de température $1.1-2.1$ oK. Méthode avec filtre superfluide.

même (fig. 4) et il faut aussi introduire un Reynolds contenant la densité totale. Ainsi, la turbulence découverte est entièrement indépendante de la vélocité du superfluide, de la vélocité relative et de l'existence ou de la non-existence du frottement mutuel.

L'analogie pour le régime turbulent avec les phénomènes en hydrodynamique classique fait suggérer la validité d'une équation de mouvement équivalente à celle de Navier-Stokes:

$$
\frac{\partial v_{n}}{\partial t}+\left(v_{n} \operatorname{grad}\right) v_{n}=-\frac{1}{\rho} \operatorname{grad} p+\frac{\eta_{n}}{\rho} \Delta v_{11}
$$

Il sera nécessaire de la réconcilier avec les équations du modèle des deux fluides $(3 a, b)$ certainement dans les deux cas étudiés par Staas et Taconis. Pour cette réconciliation une analyse simple mène à une condition qui s'écrit comme une égalité de deux expressions prises en moyenne par rapport au temps :

$$
\overline{\left(v_{n}^{t} \text { grad }\right) v_{n}^{t}}=\overline{\left(v_{s}^{t} \text { grad }\right) v_{s}^{t}}
$$

l'indice $t$ désignant la partie fluctuante de la vélocité.

Cette condition n'est pas encore comprise entièrement; une solution est naturellement l'égalité

$$
v_{n}^{t}=v_{s}^{t} \text {. }
$$

Ainsi, on pourrait faire la supposition tentante que les tourbillons dont on suppose la présence en fluide normal et en superfluide coincident. Cependant, cette question n'est pas encore éclaircie suffisamment, d'autant plus que les termes du second ordre dans les équations de mouvement $(3 a, b)$ ne sont pas encore établis d'une manière certaine. 


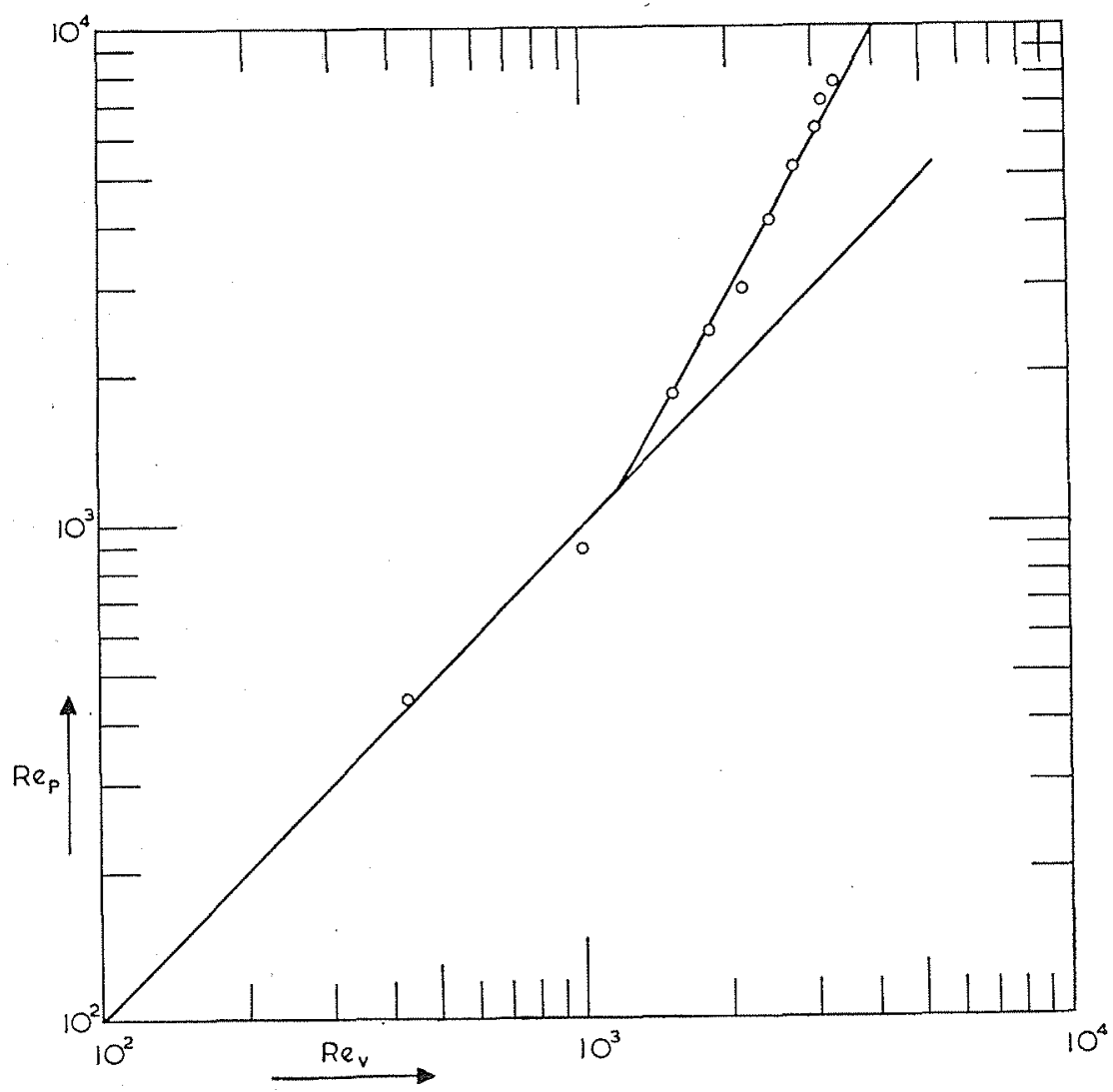

FIg. 4. - Dépendance entre $Q\left(=R e_{\mathrm{n}}\right.$ ) et le nombre de Reynolds ( $=R e_{\mathrm{V}}$ ) pour le capillaire de diamètre $255 \mu$ et la température $1,7^{\circ} \mathrm{K}$. Méthode de la "conductibilité thermique ».

Le frottement mutuel et le second son. - Une méthode évidente pour la recherche expérimentale du frottement mutuel est celle de la mesure de la " conductibilité thermique " décrite dans l'introduction. Aussi, beaucoup d'expériences ont été effectuées de cette manière, arec des conduites de géométries diverses et de dimensions jusqu'à $10^{-4} \mathrm{~mm}$. Cependant, les résultats des mesures sur des conduites plus étroites que $50 \mu$ environ sont assez problématiques. L'exposé suivant concerne seulement des tubes cylindriques d'un diamètre supérieur. Dans ce cas limité le coefficient $A$ se montre indépendant du diamètre et. s'accroit comme une fonction de la température.

Dans une méthode différente on emploje le phénomène du second son; des recherches de l'atténuation peuvent fournir de nouvelles informations. D'abord, il faut dire quelques mots sur ce phénomène très important. Il fut prédit par Tisza et Landau à la base de l'hypothèse des deux fluides et ensuite observée par Pechkov. Cette observation fut peut-être le plus grand triomphe de l'hypothèse des deux fluides.

Dans le cas du son ordinaire, on a des oscillations simultanées de la vélocité du liquide et de la densité totale; dans le cas du second son il s'agit, de même, de la vélocité relative et du rapport $\rho_{n} / \rho_{\mathrm{s}}$. L'analogue de la pression est la température et par conséquent l'instrument pour observer le phénomène est un simple thermomètre à résistance. Un élément à chauflage électrique, alimenté d'une source alternée, sert pour la génération du second son. Utilisant les équations de mouvement $(3 a, b)$ et le bilan de l'entropie on arrive à une expression pour la vélocité :

$$
v_{\mathrm{II}}^{2}=\frac{\rho_{\mathrm{S}}}{\mathrm{P}_{\mathrm{n}}} S^{2}\left(\frac{\partial T}{\partial S}\right)
$$

Cette formule permet la détermination de $p_{n}$ au moyen des mesures de la vélocité du second son.

Depuis quelque temps déjà on sait que l'atténuation du second son qu'on s'attendrait à avoir à cause des termes du frottement mutuel des équations $(3 a, b)$ ne se produit pas. Cependant, une grande atténuation se produit si l'on a un écoulement stationnaire simultané. Ces observations menèrent à l'introduction par Vinen et Hall d'une forme modifiée de la force de friction :

$$
F_{\mathrm{sn}}=A \rho_{\mathrm{n}} \rho_{\mathrm{s}}\left(\overline{v_{\mathrm{n}}-v_{\mathrm{s}}}\right)^{2}\left(v_{\mathrm{n}}-\mathrm{v}_{\mathrm{s}}\right)
$$

Ainsi la modification indique qu'on doit prendre la moyenne de la vélocité relative par rapport au temps. On peut interpréter cela par l'hypothèse que le frottement mutuel a besoin d'un certain temps pour s'établir. Vinen et Hall ont effectué des expériences sur l'atténuation du second son dans une conduite rectangulaire de quelques millimètres de largeur, la direction du second son étant perpendiculaire au courant stationnaire. Les résultats pour le coefficient $A$ s'accor- 
dent à ceux des recherches de la conductibilité thermique ». En outre la méthode nouvelle se montre d'une précision plus grande que l'autre et cela permet de faire des observations en région critique et souscritique. Cependant, cette méthode n'est convenable qu'en conduites rectangulaires très larges. C'est pour cela que Wiarda et, l'auteur ont employé une méthode par l'atténuation du second son non limatée ainsi. Le principe est montré dans la figure $5 a$ : un volume isolé est joint par une conduite au bain d'hélium. Le volume contient un élément à chauffage par le courant continu, un autre pour la génération du second son et un thermomètre pour l'observation du même. L'ensemble se comporte comme un résonateur de Helmholtz pour le second son, la fréquence étant donnée par :

$$
\nu=\frac{v_{\mathrm{II}}}{2 \pi} \sqrt{\frac{O}{l V}}
$$

Une construction différente est montrée dans la figure $5 b$.

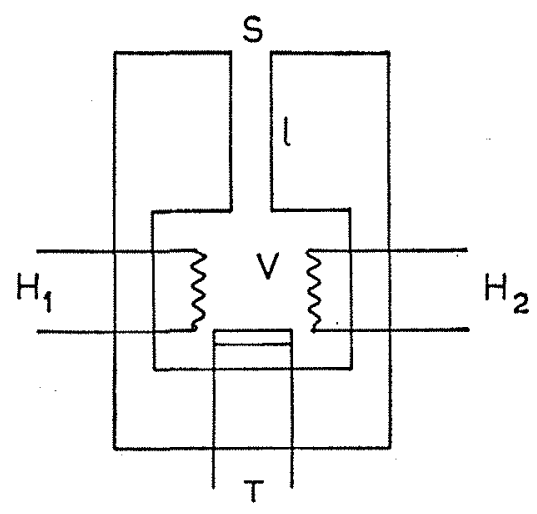

a.

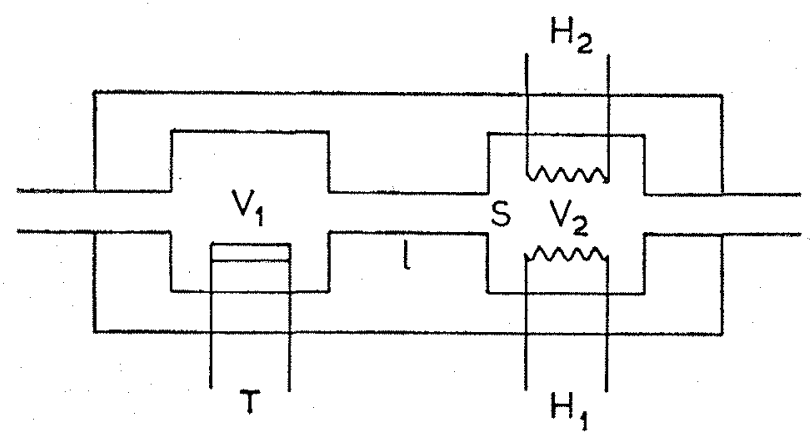

$b$.

Frg. 5. - Le principe du résonnateur de Helmholtz pour le second son.

a) Le dispositif du volume joint au bain infini.

b) Le dispositif de deux volumes "à balançoire ».

La détermination de l'atténuation est effectuée par la mesure de la largeur de la courbe de résonance, suivant une méthode bien connue.

En l'absence d'un écoulement continu l'atténuation est causée par l'action de la viscosité sur la paroi de la conduite. L'introduction d'un tel courant, donne une atténuation du caractère attendu de l'expression, c'est- à-dire, proportionnelle au carré du courant de chaleur. La figure 6 donne un exemple d'une observation. Le racine de l'atténuation liée au frottement mutuel, est

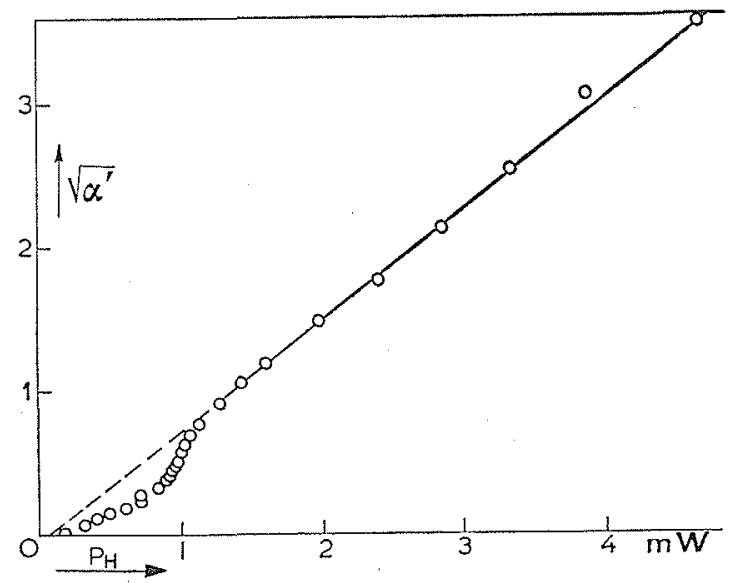

FIG. 6. - Dépendance entre la racine carrée de l'atténuation et le courant thermique continu. $T=1,5 \circ \mathrm{K}$.

représentée en fonction du courant de chaleur continu. La ligne droite correspond au frottement mutuel décrit ci-dessus.

La région critique est très évidente dans la figure. En outre, on peut noter une atténuation sous-critique, lacuelle n'est pas encore éclaircie suffisamment.

La dépendance de la température du coefficient $A$ est montrée sur la figure 7. Pour la comparaison,

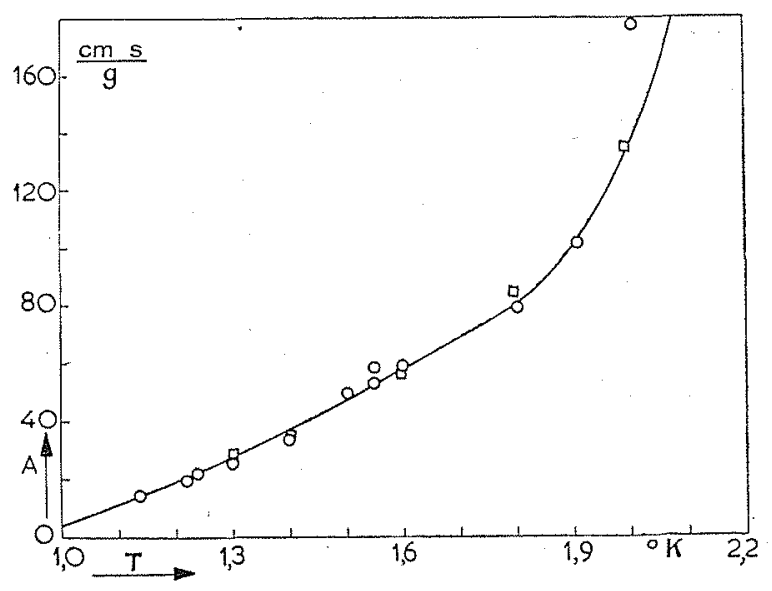

FIG. 7. - Le coefficient du frottement mutuel $A$. $\square$ Mesures de Vinen et Hall.

$\odot$ Mesures de Wiarda et Kramers.

quelques points de mesure de la recherche de Vinen sont indiqués. Toutes les méthodes expérimentales sont d'accord quant aux valeurs du coefficient $A$. Cela est particulièrement remarquable pour les études effectuées à Leyde, parce que les tubes utilisés avaient des longueurs assez petites. Dans quelques cas le rapport entre la longueur et le diamètre n'était plus que 4. La conclusion importante qu'on peut tirer de cela est que le frottement mutuel est homogène, isotrope et remplit toute la conduite. Bien que cet exposé ne s'occupe pas 
du problème de la vélocité critique, les résultats d'un seul tube sur elle sont montrés dans la figure 8. La

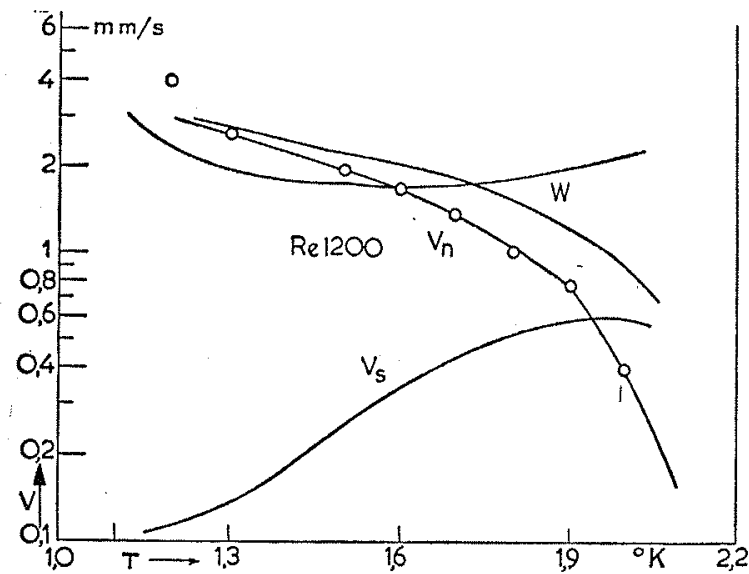

Fig. 8. - Valeurs des vélocités critiques. La ligne assez horizontales représente la valeur de $\varphi_{11}$ pour un nombre de Reynolds égal à 1.200.

Diamètre : $630 \mu$.

dépendance de la température est donnée pour les valeurs critiques de $v_{n}, v_{\mathrm{s}}$ et $w$. Pour la comparaison une ligne indique la valeur de $v_{n}$ pour une valeur fixée du nombre de Reynolds. Il est évident que la vélocité critique du frottement mutuel n'est pas du tout liée à un nombre critique de Reynolds.

Conclusion. - La nature du frottement mutuel est une question encore assez délicate. Néanmoins, une conclusion importante peut ètre tirée de l'étude du second son dont la vélocité ne change pas en présence du frottement. Par conséquent, le rapport $\rho_{\mathrm{B}} / \rho_{n}$ n'est pas influencé, et on doit conclure que le modèle des deux fluides est préservé essentiellement.

Deux écoles existent dans l'hydrodynamique de l'hélium superfluide. L'une, représentée par Vinen et Hall attribue le frottement mutuel à l'interaction entre le fluide normal ou plutôt les excitations qui le constituent, et des lignes de tourbillons se produisant dans le superfluide. Il occuperait trop de temps d'expliquer la théorie des lignes de tourbillons quantifiées (quantized vortex lines) et de décrire des expériences qui semblent la confirmer. Cependant, cette théorie donne une image très élégante de la situation, peut expliquer plus ou moins la forme du frottement (10) mais évoque beaucoup de questions irrésolues.

L'autre école, représentée par un vrai hydrodynamicien C. C. Lin de M. I. T. a critiqué l'essence microscopique de la théorie des lignes de tourbillons et a tendu à construire une théorie macroscopique. Malheu- reusement, cette théorie n'a pas encore pu fournir des prédictions qu'on puisse vérifier expérimentalement.

Un des problèmes urgents est l'insertion de la turbulence quasi-classique dans le cadre du modèle des deux fluides et sa connection avec le frottement mutuel. Sa réconciliation avec la théorie de Vinen et Hall nous semble encore assez problématique. Quoiqu'il en soit, les physiciens des basses températures ont besoin de l'aide des hydrodynamiciens pour la résolution des questions liées à ces problèmes.

\section{Discussion}

M. le Président signale l'intérêt de l'apparition de phénomènes qui ressemblent à la turbulence dans l'écoulement de l'hélium liquide car cette analogie des liquides ordinaires et des superfluides permettra peutêtre de mieux comprendre les uns et les autres.

M. le Président se demando si l'opposition entre les idées de M. Winter et de M. Kramers est profonde ou seulement apparente. Est-ce que les "particules délocalisées "ne sont pas aussi bien la "composante suprafluide"

M. Winter pense qu'il n'y a pas de contradiction si l'on admet l'existence de deux fluides : c'est, en quelque sorte, une particularisation du modèle des deux fluides.

M. le Président demande si les deux fluides sont l'un à côté de l'autre ou l'un dans l'autre.

M. Winter répond que, d'après sa théorie, ils seraient séparés.

M. Kramers rappelle qu'il a dit, au début de son exposé, qu'il considérait l'hypothèse des deux fluides comme un modèle; et le modèle est limité.

M. le Président remarque que e'est, évidemment, un modèle phénoménologique et qu'on n'a pas la prétention de faire la synthèse complète.

M. Kramers rappelle qu'avec la théorie de Landau on a un fondement, mais seulement dans le cas des termes du premier ordre. Cette théorie ne peut pas donner grand'chose pour le frottement mutuel.

M. Winter pense que, dans la théorie de Landau, les deux fluides sont mêlés, puisque les rotons se promènent partout; ce sont les rotons qui caractérisent le fluide normal.

M. Kramers indique que les phonons caractérisent aussi le fluide normal et que toute l'énergie réside dans ce dernier.

M. Winter précise que le superfluide n'a ni rotons, ni phonons, et qu'il est géométriquement séparé du fluide normal.

M. Kramers pense qu'il est très dangereux de parler en même temps des atomes, des phonons et des rotons, car il s'agit de modèles différents.

M. le Président remercie beaucoup le Dr Kramers d'avoir présenté certains aspects nouveaux de la théorie de Landau, ainsi que des expériences très intéressantes.

\section{BIBLIOGRAPHIE}

[1] Voir pour la littérature avant 1959: ATKins (K. R.), Liquid Helium, Cambridge University Press.

[2] Vinen (W. F.), Progress in Low Temp. Phys. édité par C. J. Gorter, volume III, Ch. I ; North Holland Publ. Co, 1961.

[3] Hall (H. E.), Phil. Mag., Supp. (1960), 9, 89.
[4] Staas (F. A.), Taconis (K. W.) and van Alphen (W* M.), Communications Kamerlingh Onnes Lab., Leiden no 328d; Physica (1961), 27, 893.

[5] Lin (C. C.), Phys. Reo., Letters (1959), 2, 245.

[6] LrN (C. C.), école in ternationale "Enrico Fermi ", 1961 ; publication prévue dans Nuovo Cimento. 